Volume 6, Issue 1, 1-3 Pages

Research Article | Open Access

ISSN (Online)- 2380-5706

DOI : $10.21694 / 2380-5706.21003$

\title{
A Case Report of Peripheral Cyanosis Associated with a Rare Adverse Reaction of Vaxzevria - AstraZeneca COVID-19 Vaccine
}

T. Pham Viet ${ }^{1}$, P. Thai Phuong ${ }^{1}$, T. Bui-Viet ${ }^{1}$, A. Nguyen Thi Ngoc ${ }^{1}$, H. Tran Quoc ${ }^{2}$, S. Duong Quy ${ }^{2,3,4}$

${ }^{1}$ Ninh Thuan General Hospital, Ninh Thuan province, Viet Nam.

${ }^{2}$ Lam Dong Medical College, Da Lat, Viet Nam.

${ }^{3}$ Penn State Medical College, Pennsylvania, USA.

${ }^{4}$ Pham Ngoc Thach Medical University, Ho Chi Minh city, Viet Nam.

\begin{abstract}
In the current pandemic of SARS-CoV-2, the fast development ofvaccines was mandatory due to many deaths and cases across theworld since the beginning of the outbreak. The European Medicines Agency (EMA) has allowed emergency use authorization for treatment with the Vaxzevria (ChAdOx1-S, AstraZeneca) vaccines in the European Union. There are a lot of adverse drug reactions observed during the vaccination campaign, the most common side effects are tenderness, pain and bruising at the injection site, headache, tiredness, muscle pain, general feeling of being unwell, chills, fever, joint pain and nausea (feeling sick), and can also rarely cause anaphylactic shock. The recognition of adverse drug reactions of the vaccine Vaxzevria plays an important role in minimizing vaccine-induced adverse reactions. In this report, we record and analyze a rare case of peripheral cyanosis appearing after being vaccinated, and sharing the way to resolve this problem to contribute to the data of adverse drugs reaction regarding Vaxzevria.
\end{abstract}

KEYWORDS: SARS-CoV-2 vaccine, Vaxzevria-AstraZeneca vaccine, Peripheral cyanosis, adverse drug reaction.

\section{INTRODUCTION}

The "Severe Acute Respiratory Syndrome Corona virus 2 (SARS-CoV-2)" disease has caused a challenging and threatening pandemic globally (COVID-19). This virus is highly contagious and has caused disruption of the world's health and economy. The prevalence and mortality rates of SARSCOV-2 are changing on a daily basis. According to World Health Organization (WHO) as of August 27, 2021, the COVID-19 pandemic involved 216 countries, with 214.468.601 confirmed cases including 4.470.969 death [1]. During the COVID-19 pandemic, globally people are facing major health care challenges, lockdowns, anxiety and stress, as there is no specific treatment and vaccination for this pandemic.

Given the lack of specific therapy for and the rapid spread of this virus, vaccination would be a significant tool in the fight against the SARSCoV-2 pandemic. In January 2021, the European Medicines Agency (EMA) authorized use of the viral vector coronavirus disease 2019 (COVID-19) vaccine Vaxzevria (ChAdOx1-S, AstraZeneca) in the European Union. The vaccine has been granted a conditional marketing authorisation or emergency use in more than 80 countries across six continents. More than 800 million doses of COVID-
19 Vaccine AstraZeneca have been supplied to more than 170 countries worldwide, including more than 100 countries through the COVAX Facility. Given the scale-up of mass vaccination campaigns across the world, it is likely that new adverse reactions will occur that were not reported in the initial trials. The present report aims to describe a case of Peripheral cyanosis as rare adverse drugs reactions after immunization with the viral vector vaccine Vaxzevria. [2]

\section{CASE PRESENTATION}

A men 43 years old, Vietnamese, with good health and no medical history nor allergies, received the first injection of Astrazeneca (Lot. PV46707 - intramuscular injection) on August 13th, 2021. Three days after injection, the patient discovered purple bruises on the fingertips and toes, no pain in the fingertips, and no other signs; the patient had no fever and had a stable health condition

After five days, seeing the purple bruises showed no signs of improvement; the patient went to the hospital for examination and was hospitalized at Ninh Thuan General Hospital, Ninh Thuan, Vietnam, at 17:40 on August 19th, 2021 , with the bluish of the fingers and toes extremities. Clinical examination found that the patient was in good health, subclinical results were entirely typical, platelets 
parameters and the D-dimer, Fibrinogen level was average, Sp02 was 98\%.The patient was treated with:

Methyl prednisolone 125mg, intravenous, for 3 days;

Enoxaparin $1 \mathrm{mg} / \mathrm{kg}$, Subcutaneous, for 3 days

Nifedipin 20mg, PO, 1 tablet/day, for 3 days.

Trimetazidin 35 mg, PO, 1 tablet/day, for 3 days.

Pentoxyphyllin 100mg, intravenous injection, for 3 days

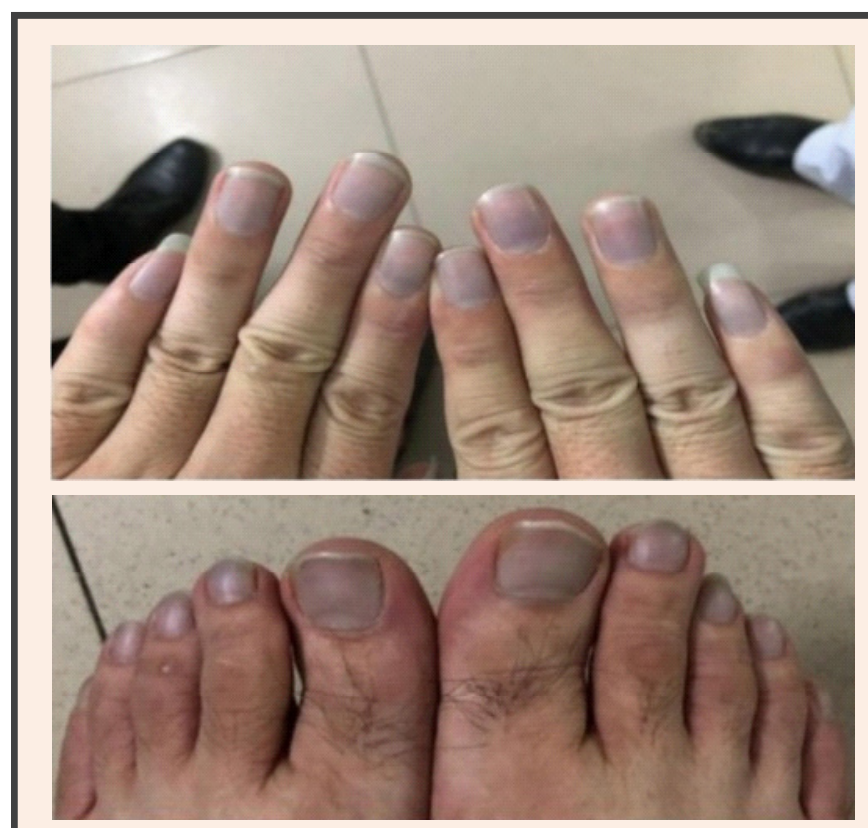

The sign of peripheral cyanosis after 3 days of vaccination

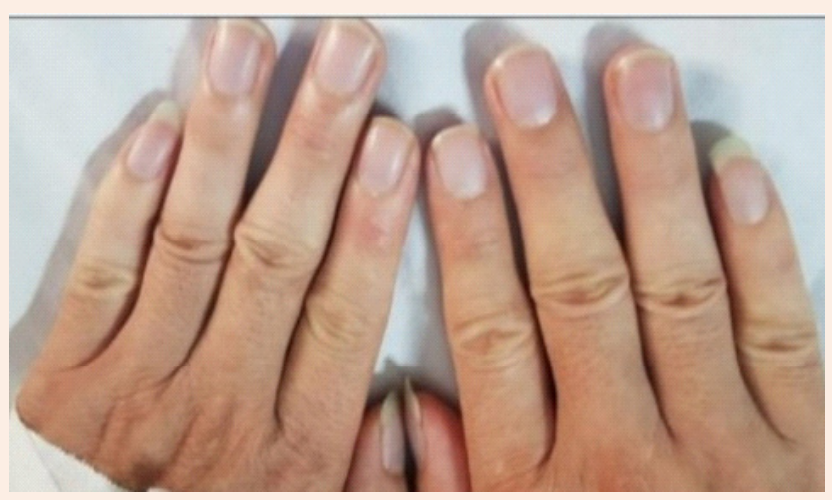

Peripheral cyanosis completely recovery after 6 days treatment

After three treatment days, the patient recovered completely without sequelae. We perform the Clinical examination with complete blood count, D-dimer and Fibrinogen, finally it showed an entirely typical result. The patient was then selfmonitored at home, and after one week of observation, the patient did not found any further abnormal signs.

\section{DISCUSSION}

\section{Adverse Drug Reactions Induced by Vaxzevia was Reported}

People receiving Vaxzevria may experience more than one side effect at the same time. The most common side effects are tenderness, pain and bruising at the injection site, headache, tiredness, muscle pain, general feeling of being unwell, chills, fever, joint pain and nausea (feeling sick). Some of side effect was observed up to 1 in 10 peoples, there are thrombocytopenia (low levels of blood platelets), vomiting, diarrhoea, pain in legs or arms, swelling and redness at the injection site, flu-like illness and asthenia (weakness). The most common side effects with Vaxzevria in the trials were usually mild or moderate and got better within a few days after vaccination. Lymphadenopathy (enlarged lymph nodes), decreased appetite, dizziness, sleepiness, lethargy (lack of energy), sweating, abdominal (belly) pain, itching, rash and urticaria (itchy rash) may affect up to 1 in 100 people.Thrombosis (formation of blood clots in the blood vessels) in combination with thrombocytopenia (thrombosis with thrombocytopenia syndrome, TTS) may affect up to 1 in 10,000 people. A minimal number of cases of angioedema, capillary leak syndrome have occurred with Vaxzevria. Allergic reactions have occurred in people receiving the vaccine, including some cases of severe allergic reactions (anaphylaxis).[3]

\section{Clinical Characteristics of Peripheral Cyanosis}

Peripheral cyanosis occurs due to the inability of the body to deliver oxygen-rich blood to the peripheral tissues. Congestive peripheral cyanosis can be caused due to the slowing of blood flow. Ischemic peripheral cyanosis occurs when vasoconstriction leads to diminished peripheral blood flow. In peripheral cyanosis, there is normal arterial oxygen saturation but increased oxygen extraction by the peripheral tissue in the capillary bed in the setting of peripheral vasoconstriction and decreased peripheral blood flow. This results in a significant difference in the saturation between the arterial and venous blood, with increased deoxygenated blood on the venous side of the capillary beds [4].

\section{Treatment}

In this case, we evaluated the results of the laboratory tests to identify and rule out Thrombosis with Thrombocytopenia Syndrome, whether or not it occurred in the patient. Results of platelet tests (platelet count, APTT, PT), D-Dimer, Fibrinogen, X-ray, Doppler to rule out possible thrombosis or vascular occlusion in the patient. All of the above results and tests helped us feel more secure about the possibility of TTS and decided to carry out supportive treatment for the patient with anticoagulants and medications helping to improve blood flow and oxygen supply to the organs and tissues.

\section{CONCLUSION}

In our adverse drug reaction case, the Vaxzevria Astrazenecainduced peripheral cyanosis involved multiple sites, including the fingers, toes, and recovered completely after six days without sequelae. It is essential to recognize the 
early signs of side effects and immediately have medical intervention to prevent the risks of thromboembolism and other complications. Healthcare providers should report unusual adverse drug reactions associated with Vaxzevria Astrazenecause.

\section{REFERENCES}

1. World Health Organization (WHO). Coronavirus disease (COVID-19) outbreak situation, https://covid19.who. int/ coronavirus 2019, August 27, 2021.
2. Vaxzevria and mRNA COVID-19 vaccines showed similar and favourable safety profiles in a population-based cohort study of over a million people, https://www. astrazeneca.com/

3. Information for the user Vaxzevria suspension for injection COVID-19 Vaccine, https://www.ema.europa. eu/

4. Das S, Maiti A. Acrocyanosis: an overview. Indian J Dermatol. 2013 Nov;58(6):417-20.

Citation: T. Pham Viet, P. Thai Phuong, et al., "A Case Report of Peripheral Cyanosis Associated with a Rare Adverse Reaction of Vaxzevria - AstraZeneca COVID-19 Vaccine”, American Research Journal of Pharmacy, Vol 6, no. 1, 2021, pp. 1-6.

Copyright (C) 2021 T. Pham Viet, P. Thai Phuong, et al., This is an open access article distributed under the Creative Commons Attribution License, which permits unrestricted use, distribution, and reproduction in any medium, provided the original work is properly cited. 